\title{
Robot-Assisted Repair of Atrial Septal Defect: A Comparison of Beating and Non-Beating Heart Surgery
}

\author{
Taeyoung Yun, M.D. ', Hakju Kim, M.D. ${ }^{2}$, Bongyeon Sohn, M.D. ', Hyoung Woo Chang, M.D., Ph.D. ', \\ Cheong Lim, M.D., Ph.D. ', Kay-Hyun Park, M.D., Ph.D. ${ }^{1}$ \\ 'Department of Thoracic and Cardiovascular Surgery, Seoul National University Bundang Hospital, Seoul National University College of Medicine, Seongnam; \\ ${ }^{2}$ Department of Thoracic and Cardiovascular Surgery, Inje University Ilsan Paik Hospital, Inje University College of Medicine, Goyang, Korea
}

\author{
ARTICLE INFO \\ Received September 24, 2021 \\ Revised November 10, 2021 \\ Accepted November 17, 2021 \\ Corresponding author \\ Cheong Lim \\ Tel 82-31-787-7134 \\ Fax 82-31-787-4050 \\ E-mail mluemoon@snubh.org \\ ORCID \\ https://orcid.org/0000-0003-0913-7014 \\ 'This study was presented in 2019 at \\ the 51st Annual Meeting of the Korean \\ Society for Thoracic and Cardiovascular \\ Surgery.
}

\begin{abstract}
Background: Robot-assisted repair of atrial septal defect (ASD) can be performed under either beating-heart or non-beating-heart conditions. However, the risk of cerebral air embolism (i.e., stroke) is a concern in the beating-heart approach. This study aimed to compare the outcomes of beating- and non-beating-heart approaches in robot-assisted ASD repair.

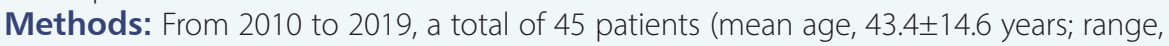
19-79 years) underwent ASD repair using the da Vinci robotic surgical system. Twenty-seven of these cases were performed on a beating heart (beating-heart group, $n=27$ ) and the other cases were performed on an arrested or fibrillating heart (non-beating-heart group, $\mathrm{n}=18$ ). Cardiopulmonary bypass (CPB) was achieved via cannulation of the femoral vessels and the right internal jugular vein in all patients.

Results: Complete ASD closure was verified using intraoperative transesophageal echocardiography in all patients. Conversion to open surgery was not performed in any cases, and there were no major complications. All patients recovered from anesthesia without any immediate postoperative neurologic symptoms. In a subgroup analysis of isolated ASD patch repair (beating-heart group: $n=22$ vs. non-beating-heart group: $n=5$ ), the operation time and CPB time were shorter in the beating-heart group ( $234 \pm 38$ vs. $253 \pm 29$ minutes, $p=0.133$ and $113 \pm 28$ vs. $143 \pm 29$ minutes, $p=0.034$, respectively).

Conclusion: Robot-assisted ASD repair can be safely performed with the beating-heart approach. No additional risk in terms of cerebral embolism was found in the beating-heart group.
\end{abstract}

Keywords: Robotic surgical procedures, Minimally invasive surgical procedures, Atrial heart septal defects

\section{Introduction}

Conventional heart surgery requires a large incision, a long hospital stay, and a prolonged recovery time [1]. Minimally invasive cardiac surgery has been successful in reducing surgical trauma and facilitating faster recovery $[1,2]$. Since the 1990s, the introduction of robotic cardiac surgery has resulted in high patient satisfaction, along with satisfactory outcomes in terms of safety and efficacy [3]. Robotic cardiac surgery is now considered acceptable as a firstchoice option over conventional surgery in light of evidence from previous studies that it has low complication and mortality rates $[2,4]$.

In our center, we have performed robotic cardiac surgery using the da Vinci Surgical System (Intuitive Surgical Inc., Sunnyvale, CA, USA) since February 2010 for conditions ranging from simple atrial septal defect (ASD) to complex cardiac disease and in procedures encompassing mitral valve repair, cardiac tumor removal, cryo-maze procedures, and minimally invasive coronary artery bypass. Kim et al. [5] reported our single-center experience in robotic cardiac surgery, with no instances of in-hospital mortality in 50 cases.

However, in robotic surgery using the traditional non- 
beating-heart approach, when the heart is arrested, performing aortic cross-clamping through a limited incision is not as easy as in the sternotomy approach [6]. Furthermore, using a root vent catheter for cardioplegia infusion and de-airing is more difficult in the robot-assisted approach $[7,8]$. The same concern exists if the heart is fibrillating, since insertion and clipping of the alligator line are technically difficult with a small, limited incision. For simple ASD repair candidates, to overcome these problems, we adopted the technique of on-pump beating-heart ASD repair without cross-clamping the aorta in 2012. In this study, we report our experience with robot-assisted ASD repair on the beating heart and compare its safety with the non-beating-heart (i.e., arrested or fibrillating heart) technique.

\section{Methods}

The study protocol was approved by the Institutional Review Board of Seoul National University Bundang Hospital as a minimal-risk retrospective study (approval no., B2107-697-106) that did not require individual consent based on the institutional guidelines for waiving consent.

\section{Patient characteristics}

We enrolled 45 consecutive patients who underwent totally robotic ASD repair using the da Vinci surgical system from February 2010 to February 2019 at Seoul National
University Bundang Hospital. The preoperative diagnosis of ASD and measurements of defect size, pulmonary-tosystemic blood flow ratio, tricuspid valve regurgitation, and pulmonary artery pressure were performed using transthoracic echocardiography (TTE). Patients were presented with either secundum or primum-type ASD. The mean size of the ASD was $25.4 \mathrm{~mm}$ (range, $10-63 \mathrm{~mm}$ ). The type and size of the ASD did not alter the surgical plan. Preoperative moderate to severe tricuspid regurgitation was confirmed preoperatively in 8 patients. The nonbeating-heart group included a total of 18 cases, of which 12 were performed on an arrested heart and 6 were performed on a fibrillating heart. The other 27 cases were beating-heart procedures (Table 1).

\section{Surgical technique}

After the induction of general anesthesia, patients were intubated with double-lumen endotracheal tubes for single-lung ventilation. A transesophageal echocardiography (TEE) probe and arterial pressure-monitoring line were inserted. A central venous catheter and a venous drainage catheter were placed percutaneously in the right internal jugular vein. External defibrillator patches were placed. Each patient was positioned with the right side of the chest elevated by $30^{\circ}$ and with the right shoulder facing up. Femoral arterial and venous cannulation was performed through a $2-\mathrm{cm}$ right inguinal cut-down incision.

After the initiation of 1-lung ventilation, a $4-\mathrm{cm}$ thora-

Table 1. Baseline characteristics of the patients

\begin{tabular}{|c|c|c|c|c|}
\hline Characteristic & Total $(\mathrm{N}=45)$ & Beating heart $(n=27)$ & Non-beating heart $(n=18)$ & $\mathrm{p}$-value \\
\hline Age $(y r)$ & $43.4 \pm 14.6$ & $43.2 \pm 16.9$ & $43.7 \pm 10.9$ & 0.835 \\
\hline Sex (male) & $27(60.0)$ & $16(59.3)$ & $11(61.1)$ & 0.901 \\
\hline Body mass index $\left(\mathrm{kg} / \mathrm{m}^{2}\right)$ & $23.2 \pm 3.1$ & $22.9 \pm 3.0$ & $23.7 \pm 3.3$ & 0.376 \\
\hline \multicolumn{5}{|l|}{ Comorbidities } \\
\hline Hypertension & $5(11.1)$ & $3(11.1)$ & $2(11.1)$ & $>0.999$ \\
\hline Atrial fibrillation & $7(15.6)$ & $2(7.4)$ & $5(27.8)$ & 0.065 \\
\hline Coronary artery disease & $1(2.2)$ & 0 & $1(5.6)$ & - \\
\hline Tricuspid regurgitation & $8(17.8)$ & $3(11.1)$ & $5(27.8)$ & 0.152 \\
\hline Congenital heart disease (except ASD) & $2(4.4)$ & 0 & $2(11.1)$ & - \\
\hline Cerebrovascular disease & $1(2.2)$ & 0 & $1(5.6)$ & - \\
\hline Diabetes mellitus & $1(2.2)$ & $1(3.7)$ & 0 & - \\
\hline Cancer $^{a)}$ & $2(4.4)$ & $1(3.7)$ & $1(5.6)$ & 0.768 \\
\hline Respiratory disease & $1(2.2)$ & $1(3.7)$ & 0 & - \\
\hline Liver disease & $2(4.4)$ & $2(7.4)$ & 0 & - \\
\hline Others & $2(4.4)$ & $2(7.4)$ & 0 & - \\
\hline ASD size (mm) & $25.4 \pm 9.1$ & $24.7 \pm 7.1$ & $26.3 \pm 11.6$ & 0.917 \\
\hline
\end{tabular}

Values are presented as mean \pm standard deviation or number (\%).

ASD, atrial septal defect.

a) Renal cell carcinoma, colon cancer. 
cotomy working window was made between the right nipple and the anterior axillary line in the fourth intercostal space. Three 8-mm endoscopic trocars for the robotic arms were placed at the anterior axillary line in the third and sixth intercostal spaces and mid-clavicular line in the fifth intercostal space, respectively. The $12-\mathrm{mm}$ endoscopic trocar was placed as a camera port via the thoracotomy working window (Fig. 1).

In the arrested-heart group, cardiopulmonary bypass $(\mathrm{CPB})$ was initiated with bicaval venous drainage. Aortic occlusion was performed with a Chitwood cross-clamp (Scanlan International Inc., St. Paul, MN, USA) through a small new incision at the posterior axillary line in the fourth intercostal space. Antegrade cold crystalloid cardioplegic solution (Custodiol HTK; Essential Pharmaceuticals, Durham, NC, USA) was administered. Ascending aorta clamping and administration of the cardioplegic solution were not performed in the fibrillating-heart and the beating-heart groups.

The intrathoracic part of the operation began with peri-

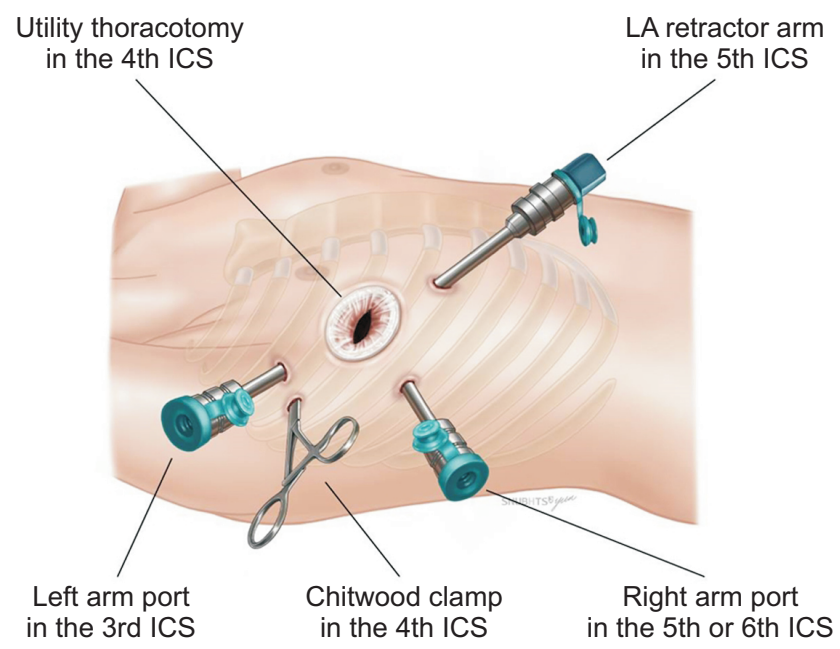

Fig. 1. Approach for robotic atrial septal defect repair using the da Vinci Surgical System. LA, left atrium; ICS, intercostal space. cardiotomy and pericardial tenting (Supplementary Video 1). After snaring of the superior and inferior vena cava, the right atrium was opened and the robotic arm retractor via the fifth intercostal space was used to expose the ASD. Depending on the size of the defect, primary repair with a running suture, a polytetrafluorourethane (PTFE) membrane (GoreTex Surgical Membrane; W.L. Gore \& Assoc, Flagstaff, AZ, USA) or a bovine pericardial patch (PeriBorn; Taewoong Medical Co. Ltd., Gimpo, Korea) was performed. These procedures were done on a beating heart in the beating-heart group (Fig. 2). To prevent air from entering the left ventricle chamber, carbon dioxide insufflation was used. The Trendelenburg position with $30^{\circ}$ left tilting was also maintained to make the atrial septum horizontal during the main procedure. Blood in the left atrial cavity was never aspirated to avoid air entrapment in the left ventricle. The rim of the ASD was exposed by gently grabbing and lifting the margin of the ASD with forceps.

For moderate to severe preoperative tricuspid regurgitation, Kay-type annuloplasty was performed. The right atriotomy was closed using a single layer of running 4-0 Gore-Tex suture with vigorous de-airing before the last closure. Air inside the heart was checked with TEE and if there was a significant amount of air inside, air aspiration was done using a $10-\mathrm{mL}$ disposable syringe with an $18 \mathrm{G}$ needle through the right pulmonary artery or vein. After the patient was weaned from CPB, complete ASD repair was confirmed by TEE. After meticulous hemostasis, the robotic arms were removed and a chest tube was inserted through the right robotic-arm port site. The right femoral artery and vein were repaired after removing the cannulae. All patients underwent TTE before discharge.

\section{Statistical analysis}

Data collection was done retrospectively. Continuous variables were expressed as mean \pm standard deviation. The Mann-Whitney U test and chi-square analysis were used to

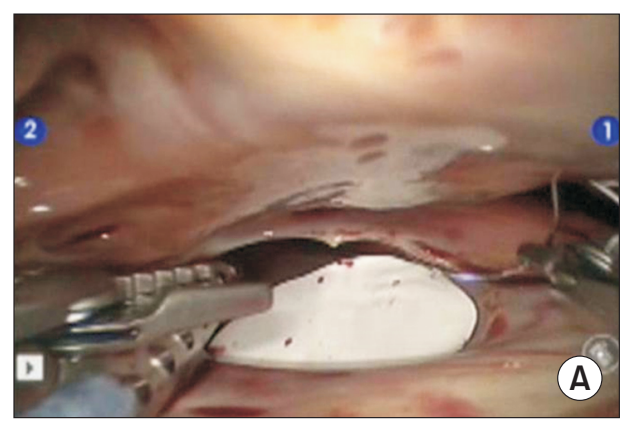

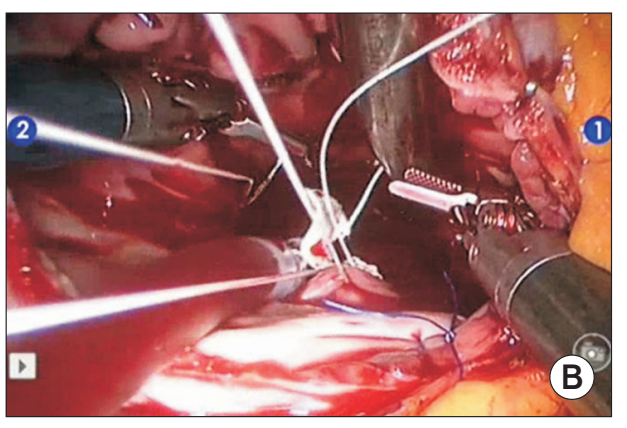

Fig. 2. Camera view in non-beating-heart $(A)$ and beating-heart conditions (B). 
compare continuous and categorical variables, respectively. The groups were stratified into the beating-heart and nonbeating-heart groups, the latter of which including arrested and fibrillating heart conditions. A subgroup analysis was done in patients who underwent isolated ASD patch closure. All statistical tests were 2-sided, with statistical significance accepted at $\mathrm{p}<0.05$. Statistical analyses were performed using IBM SPSS for Windows ver. 20.0 (IBM Corp., Armonk, NY, USA).

\section{Results}

All patients underwent successful ASD repair with the da Vinci Si surgical system. All procedures were performed by a single console surgeon (C.L.). No cases were converted to open surgery, such as thoracotomy or median sternotomy, and 1 patient underwent re-exploration for bleeding. Primary repair of ASD was done in 12 cases and patch closure was performed in 33 cases (bovine pericardial patch in 21 cases and PTFE membrane in 12 cases). Additional antithrombotic therapy with aspirin was done for the PTFE membrane patch group. Eight patients underwent ASD repair combined with tricuspid valvuloplasty, and maze procedures were performed in 5 patients.

The operative data are presented in Table 2. The operation time and $\mathrm{CPB}$ time in the beating heart group were shorter than in the non-beating heart group (234 \pm 36 versus $268 \pm 51$ minutes, $\mathrm{p}=0.021$ and $109 \pm 28$ versus $154 \pm 51$ minutes, $\mathrm{p}<0.001$, respectively). Creatinine kinase $(\mathrm{CK})$ and creatinine kinase-MB (CK-MB) serum levels were lower in the beating-heart group than in the non-beating- heart group. Specifically, the immediate postoperative CK serum levels were $1,258 \pm 897 \mathrm{IU} / \mathrm{L}$ in the beating-heart group versus $2,309 \pm 1,160 \mathrm{IU} / \mathrm{L}$ in the non-beating-heart group ( $\mathrm{p}=0.005$ ), and the CK-MB serum levels were $34 \pm 15$ versus $129 \pm 144 \mathrm{ng} / \mathrm{mL}$, respectively $(\mathrm{p}=0.013)$. The beating-heart group had shorter intensive care unit (ICU) stays than the non-beating-heart group $(2.1 \pm 0.6$ versus $2.3 \pm 0.6$ days, $\mathrm{p}=0.042$ ). However, there was no significant difference between groups in the length of hospital stay.

A subgroup analysis of isolated ASD patch repair $(n=27)$ was conducted, and the operative data are presented in $\mathrm{Ta}-$ ble 3. The operation time and CPB time were shorter in the beating-heart group $(234 \pm 38$ versus $253 \pm 29$ minutes, $\mathrm{p}=$ 0.133 and $113 \pm 28$ versus $143 \pm 29$ minutes, $p=0.034$, respectively). The immediate postoperative cardiac enzyme levels were not significantly different between these 2 groups. The length of ICU stay was not significantly different between groups $(2.0 \pm 0.3$ versus $2.1 \pm 0.6$ days, $\mathrm{p}>0.999)$. However, the length of hospital stay was longer in the beating-heart group $(8.5 \pm 2.3$ versus $6.2 \pm 0.8$ days, $\mathrm{p}=0.021)$.

All patients recovered from anesthesia without any immediate postoperative neurologic symptoms. However, 2 patients in the beating-heart group had a stroke event at postoperative days 5 and 7 , respectively. These patients had underlying atrial fibrillation. One patient experienced dysarthria, but fully recovered the day after. The other patient had right-side facial weakness with global aphasia, but had recovered by discharge. They fully recovered without sequelae. No residual ASD was detected on intraoperative TEE or postoperative TTE performed before discharge.

Table 2. Operative data

\begin{tabular}{|c|c|c|c|c|}
\hline Variable & Total $(\mathrm{N}=45)$ & Beating heart $(n=27)$ & Non-beating heart $(n=18)$ & p-value \\
\hline Combined with tricuspid valvuloplasty & $8(17.8)$ & $3(11.1)$ & $5(27.8)$ & 0.152 \\
\hline Combined with maze & $5(11.1)$ & 0 & $5(27.8)$ & - \\
\hline Combined with others & $3(6.7)$ & 0 & $3(16.7)$ & - \\
\hline Operation time (min) & $248 \pm 46$ & $234 \pm 36$ & $268 \pm 51$ & 0.021 \\
\hline CPB time (min) & $127 \pm 44$ & $109 \pm 28$ & $154 \pm 51$ & $<0.001$ \\
\hline Fibrillation time (min) & $4 \pm 12$ & 0 & $11 \pm 17$ & - \\
\hline ACC time (min) & $26 \pm 46$ & 0 & $64 \pm 53$ & - \\
\hline \multicolumn{5}{|l|}{ Immediate postoperative cardiac enzyme levels } \\
\hline CK $(I U / L)$ & $1,425(658-2,490)$ & $975(430-1,870)$ & $1,894(1,402-3,243)$ & 0.005 \\
\hline CK-MB (ng/mL) & $33(25-46)$ & $32(23-40)$ & $45(31-284)$ & 0.013 \\
\hline Troponin I (ng/mL) & $5(3-8)$ & $5(3-6)$ & $8(3-73)$ & 0.141 \\
\hline RBC transfusion (pack) & $1.0(0-2.8)$ & $1.0(1.0-3.3)$ & $5.0(0-2.0)$ & 0.114 \\
\hline Immediate postoperative neurologic symptoms & 0 & 0 & 0 & - \\
\hline Length of ICU stay (day) & $2.2 \pm 0.6$ & $2.1 \pm 0.6$ & $2.3 \pm 0.6$ & 0.042 \\
\hline Length of stay (day) & $8.6 \pm 2.9$ & $8.7 \pm 2.7$ & $8.5 \pm 3.4$ & 0.588 \\
\hline
\end{tabular}

Values are presented as number (\%), mean \pm standard deviation, or mean (range).

$\mathrm{CPB}$, cardiopulmonary bypass; $\mathrm{ACC}$, aortic cross-clamping; CK, creatinine kinase; RBC, red blood cell; ICU, intensive care unit. 
Table 3. Operative data in patients who underwent isolated ASD patch repair

\begin{tabular}{|c|c|c|c|c|}
\hline Variable & Total $(\mathrm{N}=27)$ & Beating heart $(n=22)$ & Non-beating heart $(n=5)$ & p-value \\
\hline Operation time (min) & $237 \pm 36$ & $234 \pm 38$ & $253 \pm 29$ & 0.133 \\
\hline CPB time $(\min )$ & $119 \pm 30$ & $113 \pm 28$ & $143 \pm 29$ & 0.034 \\
\hline Fibrillation time (min) & $1 \pm 7$ & 0 & $8 \pm 17$ & - \\
\hline ACC time (min) & $13 \pm 32$ & 0 & $68 \pm 46$ & - \\
\hline \multicolumn{5}{|c|}{ Immediate postoperative cardiac enzyme levels } \\
\hline CK (IU/L) & $1,239(564-1,695)$ & $1,043(520-1,608)$ & $1,494(1,387-3,413)^{a)}$ & 0.177 \\
\hline CK-MB (ng/mL) & $32(28-41)$ & $32(26-39)$ & $40(31-45)^{\mathrm{a})}$ & 0.311 \\
\hline Troponin I (ng/mL) & $5(4-6)$ & $5(4-6)$ & $3(2-8)^{\mathrm{a})}$ & 0.353 \\
\hline RBC transfusion (pack) & $1.0(1.0-2.3)$ & $1.0(1.0-2.5)$ & $2.0(0-2.5)$ & 0.950 \\
\hline Length of ICU stay (day) & $2.0 \pm 0.4$ & $2.0 \pm 0.3$ & $2.1 \pm 0.6$ & $>0.999$ \\
\hline Length of stay (day) & $8.1 \pm 2.4$ & $8.5 \pm 2.3$ & $6.2 \pm 0.8$ & 0.021 \\
\hline
\end{tabular}

Values are presented as mean \pm standard deviation or mean (range), unless otherwise stated.

ASD, atrial septal defect; CPB, cardiopulmonary bypass; ACC, aortic cross-clamping; CK, creatinine kinase; RBC, red blood cell; ICU, intensive care unit.

a)Median with range.

\section{Discussion}

The present study found no additional risk for stroke due to cerebral air embolism when robot-assisted ASD repair was performed under the beating-heart approach. The beating-heart procedure also achieved a shorter CPB time in the isolated ASD patch repair group.

Minimally invasive cardiac surgery has led to patient satisfaction with the minimal incision and faster recovery [1]. The introduction of robot-assisted surgery in the 1990s enabled totally endoscopic procedures in cardiac surgery, including coronary artery bypass, mitral valve repair, cardiac tumor removal, and ASD repair [1,7-10]. Moreover, robot-assisted endoscopic technology allowed maximum visualization of intracardiac structures by enhanced camera control $[11,12]$. Peripheral CPB has made it possible to perform totally robot-assisted cardiac surgery with a smaller incision [13]. Recent studies have evaluated the safety and efficacy of robotic cardiac surgery [1]. Seco et al. [14] reported acceptable mortality rates of $0 \%-3 \%$ in a systematic review of robotic mitral valve surgery. ASD repair can also be performed safely with robotic assistance, which was found to improve quality of life in patients undergoing surgery [3].

However, robotic procedures have a disadvantage in terms of cardioplegia administration for cardiac arrest [8]. There are several technical methods for myocardial protection in minimally invasive surgery [15]. The endoaortic balloon clamping technique, introduced by Lebon et al. [16], is a simple and feasible procedure, but has a high cost and poses a risk of aortic dissection. The second is retrograde cardioplegia infusion through the coronary sinus.
The main concern of this technique is coronary sinus rupture and inadequate myocardial protection of the right ventricle. The last is direct cardioplegia infusion into the ascending aorta, similar to conventional surgery. Direct cardioplegia infusion via the aortic root cannula was our strategy for arrested heart robotic surgery. However, when performed by an inexperienced surgeon, this technique could be technically demanding, with a higher risk of bleeding $[8,15]$. Under a fibrillating heart, the application of fibrillating wires onto the epicardium is technically demanding due to the small incision of robotic cardiac surgery.

In 2012, we started to perform beating heart ASD repair without cross-clamping the aorta or fibrillating the heart. Several reports have described surgical procedures on the beating heart in which aortic cross-clamping and cardioplegic arrest were not utilized $[9,10]$. Xiao et al. [12] argued for the safety of this technique using long-term 7-year results, with the largest group to date (160 patients).

There is a paucity of data comparing postoperative outcomes between the beating and non-beating heart approach in robotic ASD repair. In previous studies, the suggested advantage of beating heart surgery include faster recovery, myocardial protection, and shorter $\mathrm{CPB}$ and total operation times [12]. In our study, the mean operation time and CPB duration were shorter in the isolated ASD patch repair-beating heart group (but without statistical significance for $\mathrm{CPB}$ duration). These results suggest that the determination of statistical significance might have been limited by the small number of cases. Nevertheless, a higher risk of morbidity was not found in the beating heart group compared to the non-beating heart group. 
Potential concerns regarding the beating heart procedure include the risk of air embolism. To prevent this, we used carbon dioxide to insufflate the chest [12]. Blood in the left atrial cavity was not aspirated to prevent air entrapment in the left ventricle [6]. The heart was vigorously de-aired after the main surgical procedure. There were no immediate neurologic symptoms or adverse events in the patients in the beating-heart group. However, 2 patients with underlying atrial fibrillation experienced stroke on postoperative days 5 and 7, respectively, and fully recovered by discharge.

The primary limitations of this study are its retrospective, single-institutional design and the fact that it included a small number of patients. This might have led to weak statistical power. A larger study population and longer follow-up data are needed.

In conclusion, robot-assisted ASD repair can be safely performed with the beating-heart approach without an increased risk for cerebral air embolism compared to the non-beating-heart approach. Therefore, the beating-heart approach could be an option to simplify the procedure of robot-assisted ASD repair.

\section{Conflict of interest}

No potential conflict of interest relevant to this article was reported.

\section{ORCID}

Taeyoung Yun: https://orcid.org/0000-0002-4303-8493

Hakju Kim: https://orcid.org/0000-0003-0830-3287

Bongyeon Sohn: https://orcid.org/0000-0003-0613-8273

Hyoung Woo Chang: https://orcid.org/0000-0001-5516-0333

Cheong Lim: https://orcid.org/0000-0003-0913-7014

Kay-Hyun Park: https://orcid.org/0000-0002-1111-9627

\section{Supplementary materials}

Supplementary materials can be found via https://doi. org/10.5090/jcs.21.111. Supplementary Video 1. Robot-assisted repair of atrial septal defect.

\section{References}

1. Doulamis IP, Spartalis E, Machairas N, et al. The role of robotics in cardiac surgery: a systematic review. J Robot Surg 2019;13:41-52.

2. Yanagawa F, Perez M, Bell T, Grim R, Martin J, Ahuja V. Critical outcomes in nonrobotic vs robotic-assisted cardiac surgery. JAMA Surg 2015;150:771-7.

3. Morgan JA, Peacock JC, Kohmoto T, et al. Robotic techniques improve quality of life in patients undergoing atrial septal defect repair. Ann Thorac Surg 2004;77:1328-33.

4. Deeba S, Aggarwal R, Sains P, et al. Cardiac robotics: a review and St. Mary's experience. Int J Med Robot 2006;2:16-20.

5. Kim ER, Lim C, Kim DJ, Kim JS, Park KH. Robot-assisted cardiac surgery using the da Vinci surgical system: a single center experience. Korean J Thorac Cardiovasc Surg 2015;48:99-104.

6. Thapmongkol S, Sayasathid J, Methrujpanont J, Namchaisiri J. Beating heart as an alternative for closure of secundum atrial septal defect. Asian Cardiovasc Thorac Ann 2012;20:141-5.

7. Cheng Y, Chen H, Mohl W, Liu X, Si Z. Totally endoscopic congenital heart surgery compared with the traditional heart operation in children. Wien Klin Wochenschr 2013;125:704-8.

8. Watanabe G, Ishikawa N. Alternative method for cardioplegia delivery during totally endoscopic robotic intracardiac surgery. Ann Thorac Surg 2014;98:1129-31

9. Kim JE, Jung SH, Kim GS, et al. Surgical outcomes of congenital atrial septal defect using da VinciTM surgical robot system. Korean J Thorac Cardiovasc Surg 2013;46:93-7.

10. Ma ZS, Wang JT, Dong MF, Chai SD, Wang LX. Thoracoscopic closure of ventricular septal defect in young children: technical challenges and solutions. Eur J Cardiothorac Surg 2012;42:976-9.

11. Ma ZS, Dong MF, Yin QY, Feng ZY, Wang LX. Totally thoracoscopic repair of atrial septal defect without robotic assistance: a single-center experience. J Thorac Cardiovasc Surg 2011;141:1380-3.

12. Xiao C, Gao C, Yang M, et al. Totally robotic atrial septal defect closure: 7-year single-institution experience and follow-up. Interact Cardiovasc Thorac Surg 2014;19:933-7.

13. Argenziano M, Oz MC, Kohmoto T, et al. Totally endoscopic atrial septal defect repair with robotic assistance. Circulation 2003;108 Suppl 1:II191-4.

14. Seco M, Cao C, Modi P, et al. Systematic review of robotic minimally invasive mitral valve surgery. Ann Cardiothorac Surg 2013;2:70416.

15. Bainbridge D, Fernandes P, Chu MW. Cardioplegia during minimally invasive mitral valve surgery, a team approach. J Cardiothorac Vasc Anesth 2018;32:664-5.

16. Lebon JS, Couture P, Fortier A, et al. Myocardial protection in mitral valve surgery: comparison between minimally invasive approach and standard sternotomy. J Cardiothorac Vasc Anesth 2018;32:656-63. 